\title{
Solar flare soft X-ray spectra from Diogeness observations
}

\author{
Marek Stȩślicki ${ }^{1}$, Janusz Sylwester ${ }^{1}$, Barbara Sylwester ${ }^{1}$, Żaneta \\ Szaforz $^{1,2}$, Zbigniew Kordylewski ${ }^{1}$, Stefan Płocieniak ${ }^{1}$, Marek \\ Siarkowski ${ }^{1}$ and Kenneth J. H. Phillips ${ }^{3}$ \\ ${ }^{1}$ Solar Physics Division, Space Research Centre Polish Academy of Sciences \\ Kopernika 11, 51-622 Wrocław, Poland \\ email: sm@cbk.pan.wroc.pl \\ ${ }^{2}$ Astronomical Institute, University of Wroclaw \\ Kopernika 11, 51-622 Wrocław, Poland \\ email: zs@cbk.pan.wroc.pl \\ ${ }^{3}$ Earth Sciences Department, Natural History Museum \\ London, United Kingdom. \\ email: kennethjhphillips@yahoo.com
}

\begin{abstract}
Diogeness was an uncollimated scanning flat crystal spectrometer observing solar flare X-ray spectra in four narrow wavelength bands in the vicinity of Ca XIX, S XV and Si XIII He-like line 'triplets' around $3.18 \AA, 5.04 \AA$ and $6.65 \AA$. In two of the spectral channels, emission lines around the Ca XIX $3.178 \AA$ resonance line were scanned in opposite directions, being diffracted from precisely adjusted identical Quartz crystals mounted on a common shaft in a so-called Dopplerometer (tachometer) configuration. Observations of solar X-ray spectra made by Diogeness provide a direct diagnostic information on plasma characteristics during the impulsive flare energy release. We present a sample of events which occurred during the Diogeness operation time from August 16, 2001 to September 17, 2001.
\end{abstract}

Keywords. techniques: spectroscopic - Sun: corona, Sun: flares - Sun: X-rays, gamma rays

\section{Introduction}

In order to obtain a full understanding of the energetics and physical characteristics of solar active regions and the mechanism of flare energy release, reliable measurements of emitted spectra are necessary. For many years crystal spectrometers have been used to obtain soft X-ray spectra of solar emitting regions. The resulting spectra have helped our understanding of the energies and plasma characteristics of the emitting plasma. Additionally, instruments with an accurate absolute intensity calibration have enabled coronal element abundances to be determined. Unfortunately, spectra from crystal spectrometers may sometimes be subject to a confusion of Doppler and spatial shifts, especially during the initial phases of flares, without any means of disentangling the two. This difficulty can be overcome by simultaneous observations using pairs of crystals oriented in such way that the spatial and Doppler displacement shifts are in opposite directions - the "Dopplerometer" mode; a full description is given by (Sylwester et al., 2015).

\section{The Diogeness instrument}

Details of the Diogeness instrument construction have been given by Płocieniak et al. (2002) and Siarkowski et al. (2002). Diogeness had four channels covering wavelength ranges around intense resonance lines of He-like ions of silicon, sulphur, and calcium 
Table 1. Diogeness instrument channels.

\begin{tabular}{lcccc}
\hline Channel & $\mathbf{1}$ & $\mathbf{2}$ & $\mathbf{3}$ & $\mathbf{4}$ \\
\hline Crystal & Quartz & ADP & Beryl & Quartz \\
Wavelength range $(\AA)$ & $3.1436-3.3915$ & $4.9807-5.3721$ & $6.1126-6.7335$ & $2.9601-3.2123$ \\
Observed principal lines & Ca XIX & S XV & Si XIII & Ca XIX \\
\hline
\end{tabular}
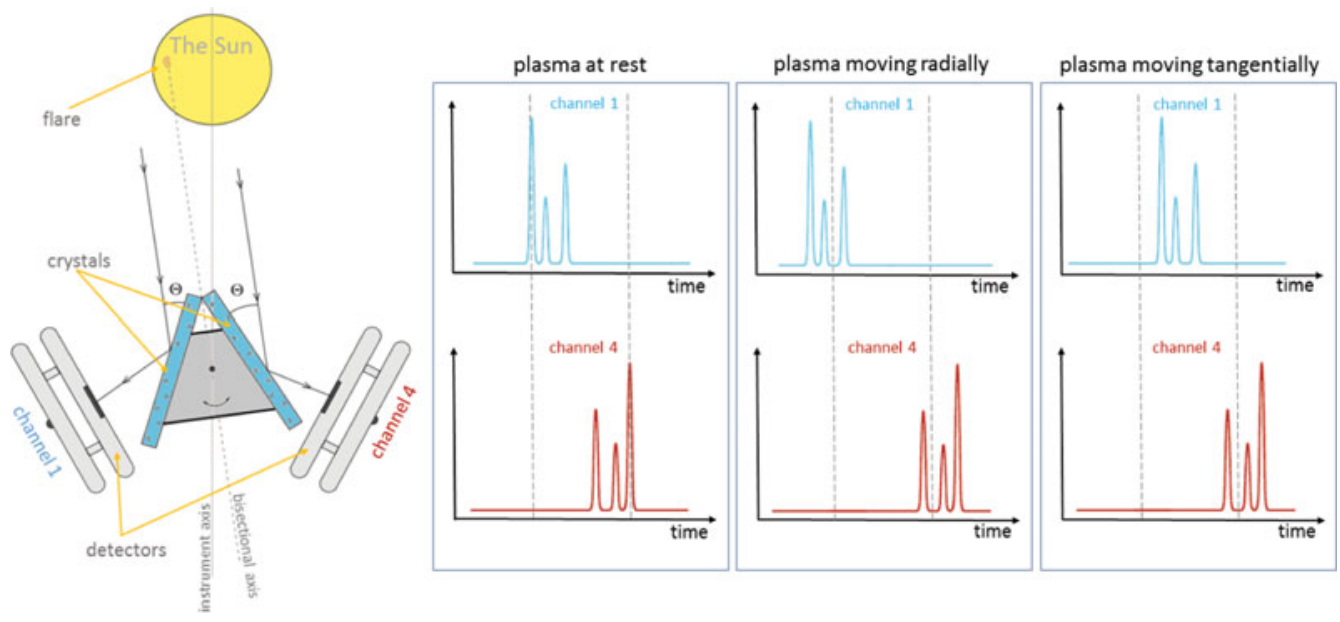

Figure 1. The principle of the Diogeness dopplerometer concept. Left: Solar X-rays are incident on two identical quartz crystals oriented as shown. The diffracted photons are detected by proportional counters (labelled 1 and 4). Right panels: The resulting spectra. In the left panel the flare plasma is assumed to be at rest and the lines are coincident (for detector 1 wavelength increases to the right, for detector 4 in opposite direction). In the center panel flare plasma is assumed to be in radial motion with respect to the instrument and the lines are shifted in opposite directions. In the right: any tangential motions along crystal dispersion direction are observed as a shifts in identical directions.

emitted during solar flares. Two quartz crystals covered the Ca XIX lines (channels 1 and 4), and ADP and beryl crystals (channels 2 and 3) covered the S XV and Si XIII lines respectively (Table 1 ).

Flat mono-crystals were mounted on a common rotatable shaft, so back-and-forth scanning motions of the shaft allowed spectra to be viewed. Silicon and sulphur lines (channels 2 and 3) were scanned repeatedly during the development of flares in the direction of alternately increasing and decreasing wavelengths. Figure 1 shows the arrangement for channels 1 and 4 with an identical pair of quartz crystals (cut from the same mono-crystal) oriented in the dopplerometer configuration.

\section{Diogeness observations}

The Diogeness instrument had a lifetime of only a few weeks, but during that time spectra from nine substantial flares with GOES classes ranging from $\mathrm{C}$ to $\mathrm{X}$ were recorded. Table 2 gives details of these flares including times, standard IAU flare notation, GOES and $\mathrm{H} \alpha$ importance, and heliographic locations. More than 200 spectra were obtained in the four channels for these events. Example spectra for the M1.5-class flare on August 30, 2001 are shown in Figure 2. 
Table 2. Solar flares observed by Diogeness.

\begin{tabular}{lccc}
\hline Flare & GOES & H $\alpha$ & Location \\
\hline SOL2001-08-25T09:28 & M1.2 & - & S18E31 \\
SOL2001-08-25T16:45 & X5.3 & 3B & S17E34 \\
SOL2001-08-30T17:57 & M1.5 & 2N & S21W28 \\
SOL2001-09-02T06:02 & M1.3 & 1F & S17W66 \\
SOL2001-09-02T13:48 & M3.0 & 2N & S21W56 \\
SOL2001-09-03T01:58 & C9.0 & - & $\sim$ E90S15 \\
SOL2001-09-03T17:16 & M1.1 & - & N10W06 \\
SOL2001-09-03T18:41 & M2.5 & - & $\sim$ E90S28 \\
SOL2001-09-16T03:53 & M5.6 & 2N & S29W54 \\
\hline
\end{tabular}
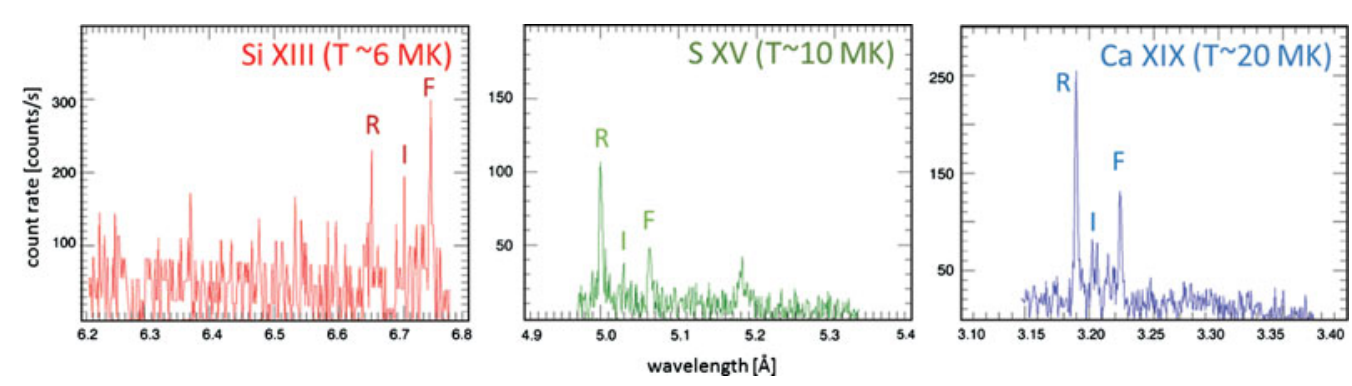

Figure 2. Spectra during the M1.5 class solar flare from August 30, 2001 observed at 17:52 UT by three of the Diogeness channels. In each channel, triplet lines emitted by He-like S, Si, and $\mathrm{Ca}$ are observed (they are labelled resonance $-\mathrm{R}$, intercombination $-\mathrm{I}$ and forbidden $-\mathrm{F}$ ). The temperatures at which lines are formed are also indicated. For measurements of Si XIII spectral range, the Beryl crystal has been used. Beryl $\left(\mathrm{Be}_{4} \mathrm{Al}_{2} \mathrm{Si}_{6} \mathrm{O}_{18}\right)$ incorporate Si atoms in the crystal lattice and therefore its X-ray reflectivity has absorption jump just left to the forbidden line $(\mathrm{F})$. This causes that on the recorded spectrum the intensity of $\mathrm{R}$ line is less than the $\mathrm{F}$ line. This effect will be corrected on the Level_2 reduced spectra.

\section{Summary}

Some 200 high-resolution X-ray spectra have been obtained with the Diogeness instrument during nine large solar flares in 2001. Analysis is proceeding of these spectra which cover the triplet of lines due to He-like $\mathrm{S}, \mathrm{Si}$, and $\mathrm{Ca}$ ions to give temperature and other information about the emitting plasmas. Of particular interest are the Ca XIX spectra in the dopplerometer mode which will allow Doppler and spatial shifts to be disentangled. The same principle will be used for the ChemiX instrument which will be on board the forthcoming Interhelioprobe Mission (Sylwester et al., 2015).

\section{Acknowledgements}

We acknowledge financial support from the Polish National Science Centre grant number 2011/01/M/ST9/06096, 2011/01/M/ST9/05878 and 2013/11/B/ST9/00234.

\section{References}

Płocieniak, S., Sylwester, J., \& Kordylewski, Z., et al. 2002, ESA SP, 506, 963

Siarkowski, M., Sylwester, J., \& Płocieniak, S. et al. 2002, ESA SP, 506, 753

Sylwester, J., Kordylewski, Z., \& Płocieniak, S. et al. 2015, Solar Physics, 239, 149 